\title{
Mesobiliverdin IX $\alpha$ enhances rat pancreatic islet yield and function
}

\author{
Taihei Ito ${ }^{1}$, Dong Chen ${ }^{2,3}$, Cheng-Wei Tom Chang ${ }^{2,4}$, Takashi Kenmochi $^{1}$, Tomonori Saito ${ }^{5}$, Satoshi Suzuki ${ }^{6}$ \\ and Jon Y. Takemoto ${ }^{2,7 *}$
}

${ }^{1}$ Department of Organ Transplant Surgery, School of Medicine, Fujita Health University, Toyoake, Aichi, Japan

2 Synthetic Bioproducts Center, Utah State University, Logan, UT, USA

${ }^{3}$ Department of Biological Engineering, Utah State University, Logan, UT, USA

${ }^{4}$ Department of Chemistry and Biochemistry, Utah State University, Logan, UT, USA

${ }^{5}$ Department of Clinical Research Center, Chiba-East National Hospital, National Hospital Organization, Chiba City, Chiba, Japan

${ }^{6}$ Human and Animal Bridging Research Organization Research Laboratories, Ichikawa General Hospital, Ichikawa, Chiba, Japan

${ }^{7}$ Department of Biology, Utah State University, Logan, UT, USA

\section{Edited by:}

Momir Mikov, University of Otago,

New Zealand

\section{Reviewed by:}

Andrei Adrian TICA, University of

Medicine Craiova Romania, Romania

Rajgopal Govindarajan, University of

Georgia, Georgia

${ }^{*}$ Correspondence:

Jon Y. Takemoto, Department of Biology, Utah State University, 5305 Old Main Hill, Logan, UT 84322, USA. e-mail:jon.takemoto@usu.edu
The aims of this study were to produce mesobiliverdin IX $\alpha$, an analog of anti-inflammatory biliverdin $\mid X \alpha$, and to test its ability to enhance rat pancreatic islet yield for allograft transplantation into diabetic recipients. Mesobiliverdin IX $\alpha$ was synthesized from phycocyanobilin derived from cyanobacteria, and its identity and purity were analyzed by chromatographic and spectroscopic methods. Mesobiliverdin IX $\alpha$ was a substrate for human $\mathrm{NADPH}$ biliverdin reductase. Excised Lewis rat pancreata infused with mesobiliverdin IX $\alpha$ and biliverdin $\mathrm{IX}-\mathrm{HCl}(1-100 \mu \mathrm{M})$ yielded islet equivalents as high as 86.7 and $36.5 \%$, respectively, above those from non-treated controls, and the islets showed a high degree of viability based on dithizone staining. When transplanted into livers of streptozotocininduced diabetic rats, islets from pancreata infused with mesobiliverdin IX $\alpha$ lowered non-fasting blood glucose (BG) levels in $55.6 \%$ of the recipients and in $22.2 \%$ of control recipients. In intravenous glucose tolerance tests, fasting BG levels of 56 post-operative day recipients with islets from mesobiliverdin IX $\alpha$ infused pancreata were lower than those for controls and showed responses that indicate recovery of insulin-dependent function. In conclusion, mesobiliverdin IX $\alpha$ infusion of pancreata enhanced yields of functional islets capable of reversing insulin dysfunction in diabetic recipients. Since its production is scalable, mesobiliverdin IX $\alpha$ has clinical potential as a protectant of pancreatic islets for allograft transplantation.

Keywords: mesobiliverdin, biliverdin, pancreatic islets, islet transplantation, anti-inflammatory

\section{INTRODUCTION}

The bile pigments bilirubin (Figure 1A) and biliverdin (Figure 1B) are best known as heme degradative intermediates associated with erythrocyte and hemoglobin turnover (McDonagh, 2001). They result from ring cleavage of heme catalyzed by heme oxygenase (HO) that occurs selectively at the $\alpha$-methene bridge to generate the IX $\alpha$ isomer of biliverdin. Biliverdin IX $\alpha$ is subsequently reduced via NADPH/NADH biliverdin reductase to form the IX $\alpha$ isomer of bilirubin that in turn is consecutively bound to serum albumin and glucuronic acid for excretion in bile. The overall process serves to eliminate heme - which is toxic when accumulated.

Biliverdin IX $\alpha$ and bilirubin IX $\alpha$ are also cytoprotectants (Stocker et al., 1987; Sedlak and Snyder, 2004; Soares and Bach, 2009; Kapitulnik and Maines, 2012). Bilirubin IX $\alpha$ is well known to quench the propagation of reactive oxygen species (ROS) and consequently to confer protection against cellular oxidative damage. Biliverdin IX $\alpha$ is less appreciated as an anti-oxidant but equally effective (Stocker et al., 1987), and examples of its cytoprotective capabilities are accumulating (Nakao et al., 2004, 2005; Yamashita et al., 2004; Overhaus et al., 2006; Fujii et al., 2010; Bellner et al., 2011). The cytoprotective effects of biliverdin IX $\alpha$ also result from interaction with biliverdin reductase that plays a pivotal role in multiple downstream pathways related to cell survival and stress responses (Gibbs and Maines, 2007; Gibbs et al., 2012; Wegiel and Otterbein, 2012). Examples of biliverdin reductase mediated cytoprotective pathways are PI3K/Akt pathway-dependent protection against hypoxia/reoxygenation (Pachori et al., 2007), regulation of anti-apoptotic transcription factor NF-kB (Gibbs and Maines, 2007), induction of anti-inflammatory cytokine interferon-10 (Wegiel et al., 2009) and the nitrosylation-dependent inhibition of pro-inflammatory TLR4 expression (Wegiel and Otterbein, 2012). Thus, biliverdin IX $\alpha$ is increasingly recognized as a potential antiinflammatory therapeutic agent (Florczyk et al., 2008; Wang et al., 2011; Gibbs et al., 2012; Wegiel and Otterbein, 2012). Examples of its potential use are for ischemia/reperfusion injury following liver (Fondevila et al., 2003; Nakao et al., 2004), small bowel (Nakao et al., 2004), cardiac, renal (Nakao et al., 2005), and lung (Zhou et al., 2011) transplants, vascular injury (Ollinger et al., 2005), endotoxic shock (Sarady-Andrews et al., 2005), vascular 


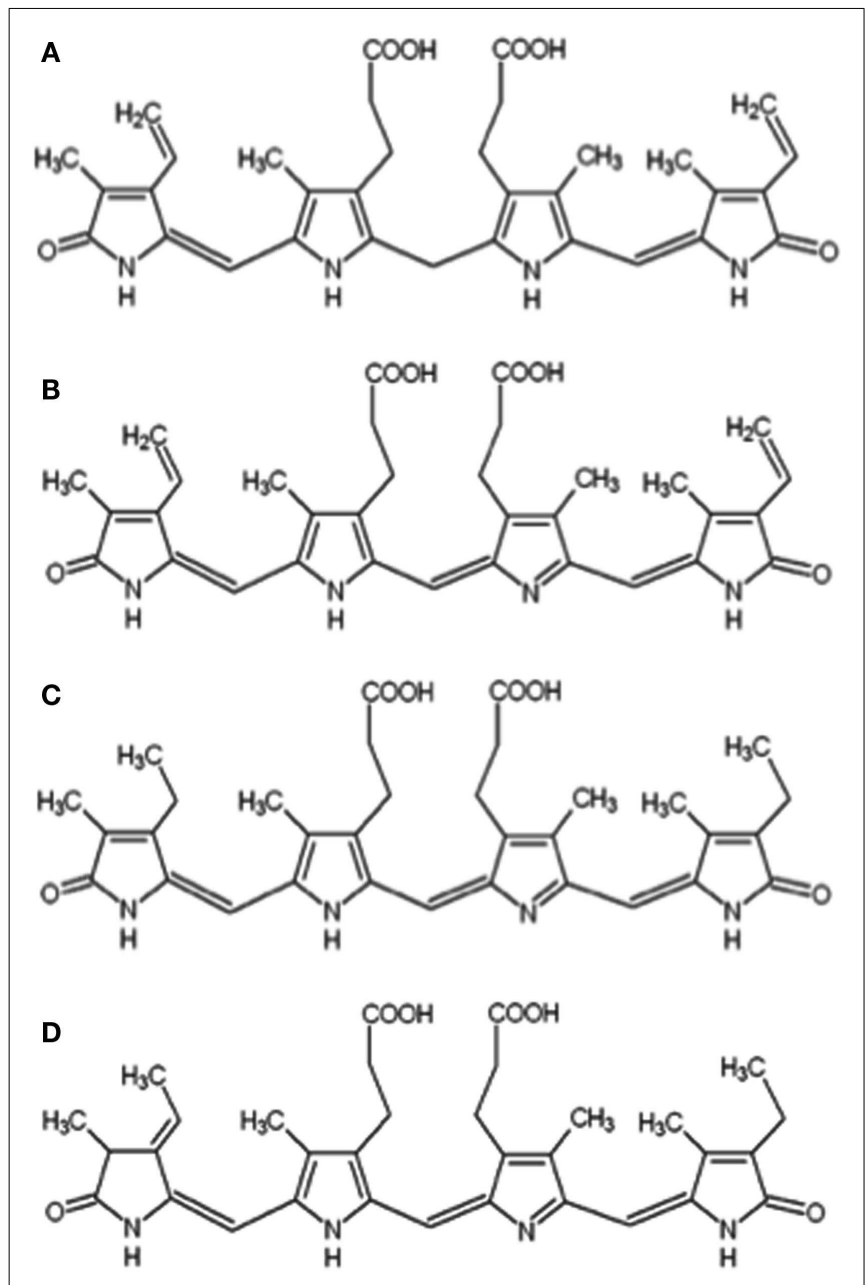

FIGURE 1 | Chemical structures of bilirubin IX $\alpha$ (A), biliverdin IX $\alpha$ (B), mesobiliverdin IX $\alpha$ (C), and phycocyanobilin (D).

intimal hyperplasia (Nakao et al., 2005), nephropathy (Fujii et al., 2010), infection by hepatitis C (Zhu et al., 2010) and other viruses (Nakagami et al., 1992; McPhee et al., 1996), and reversal of type 2 diabetes by diets supplemented with biliverdin IX $\alpha$ (Ikeda et al., 2011). Barriers to the therapeutic use of biliverdin IX $\alpha$ are limited availability, uncertain purity of commercial preparations and derivation from mammalian materials (McDonagh, 2005) prompting attempts to substitute other bile pigments to achieve therapeutic effects (Zheng et al., 2012).

Another possible application for biliverdin IX $\alpha$ is improvement of pancreatic islet allograft transplantation efficacy (Najarian et al., 1977; Matsumoto et al., 2007). In this procedure, normal islets are excised from donor pancreata, preserved in solution, and injected into the intraportal ducts of type 1 diabetic recipients leading to insulin independence and hypoglycemia awareness. The procedure is historically hindered by allograft rejection and oxidative damage of islet beta cells. Immunosuppressive strategies have lowered islet rejection rates (Shapiro et al., 2000; Ryan et al., 2004; Matsumoto et al., 2007; Kenmochi et al., 2008), but the procedure is still hampered by oxidative-stress induced apoptosis that reduces the number of transplanted islets (Emamaullee and Shapiro, 2006; Wang et al., 2011). Anti-inflammatory strategies that improve the number of effective transplanted islets include stimulation of $\mathrm{HO}$ expression (Ribeiro et al., 2003), bilirubin IX $\alpha$ administration to recipient or donor islets during processing (Wang et al., 2011), and administration of p38 MAPK inhibitor to donor pancreata (Ito et al., 2008). Biliverdin IX $\alpha$ as an anti-inflammatory islet protectant has not yet been reported due at least partly to the limited amounts of commercially available biliverdin IX $\alpha$.

Here we report the production of mesobiliverdin IX $\alpha$ (Figure 1C), a close analog of biliverdin IX $\alpha$, and determination of its ability to protect islets. Mesobiliverdin IX $\alpha$ occurs naturally in non-vertebrates and mammals, and in the latter from bacterial transformations of non-conjugated bilirubin (Greenberg et al., 1971; Tiribelli and Ostrow, 2005; Vitek et al., 2006). Mesobiliverdin IX $\alpha$ and biliverdin IX $\alpha$ share important structural features (e.g., bridging propionate groups) that permit similar substrate interaction with biliverdin reductase (Cunningham et al., 2000; Fu et al., 2012) and suggesting similar cytoprotective capabilities against cellular damage by ROS. Importantly, the described method for mesobiliverdin IX $\alpha$ production is scalable and uses an abundant non-animal source feedstock - cyanobacteria. Finally, we show the abilities of the produced mesobiliverdin IX $\alpha$ as well as biliverdin IX $\alpha-\mathrm{HCl}$ to protect pancreatic islet preparations for allograft transplantation.

\section{MATERIALS AND METHODS \\ MESOBILIVERDIN IX $\alpha$}

Mesobiliverdin IX $\alpha$ was produced from the phycocyanin chromophore, phycocyanobilin (Figure 1D), recovered from lyophilized powders of the cyanobacterium Spirulina platensis. Phycocyanin was obtained by adding $160 \mathrm{~g}$ of Spirulina powder (Bio-Alternatives, Oregon, USA) to $2 \mathrm{~L}$ water, shaking the mixture on a rotary shaker overnight $(16 \mathrm{~h})$ at $200 \mathrm{rpm}$ and $37^{\circ} \mathrm{C}$, and centrifuging $(90 \mathrm{~min}, 1597 \times \mathrm{g})$ the mixture at $4^{\circ} \mathrm{C}$. The supernatant fluid was recovered and $530 \mathrm{~g}$ of $\left(\mathrm{NH}_{4}\right)_{2} \mathrm{SO}_{4}$ was slowly added with stirring to give a $50 \%$ saturated solution. The solution was incubated in ice water for $30 \mathrm{~min}$. After centrifugation $(15971 \times \mathrm{g}$, $30 \mathrm{~min}$ ), the dark-blue phycocyanin was collected and washed with $700 \mathrm{~mL}$ methanol. The centrifugation and washing (with $300 \mathrm{~mL}$ methanol) was repeated four times. Phycocyanobilin was obtained by cleavage of thioether bonds between the bile pigment and phycocyanin apoprotein. Washed phycocyanin generated from $160 \mathrm{~g}$ Spirulina powder was added to $600 \mathrm{~mL}$ methanol and reflux boiled with stirring for $16 \mathrm{~h}$. After centrifugation at $6371 \times g$ for $5 \mathrm{~min}$, the supernatant fluid containing phycocyanobilin was recovered and concentrated to $\sim 40 \mathrm{~mL}$ by rotary evaporation. The concentrated phycocyanobilin solution was mixed with $25 \mathrm{~mL}$ chloroform and the mixture added to and shaken with $200 \mathrm{~mL}$ purified water (previously acidified with $300 \mu \mathrm{L} 0.5 \mathrm{~N} \mathrm{HCl}$ ) in a separatory funnel. Phycocyanobilin was recovered in the chloroform layer. The pigment extraction was repeated three times with $10 \mathrm{~mL}$ volumes of chloroform. The chloroform fractions were combined and reduced to $\sim 10 \mathrm{~mL}$ by evaporation with nitrogen gas. The reduced pigment solution was added to $60 \mathrm{~mL}$ hexane and centrifuged for $3 \mathrm{~min}$ at $4500 \times g$ and the pigmented pellet was air-dried. Typical yields were $\sim 100 \mathrm{mg}$ phycocyanobilin $160 / \mathrm{g}$ Spirulina powder. 
Phycocyanobilin $(180 \mathrm{mg}$ ) was added to $40 \mathrm{ml}$ methanol with $400 \mathrm{mg} \mathrm{K} \mathrm{CO}_{3}(10 \mathrm{mg} / \mathrm{mL})$ and $400 \mathrm{mg} \mathrm{NaHCO}$. After boiling under reflux for $8 \mathrm{~h}$, the solution was added to $200 \mathrm{~mL}$ water. Mesobiliverdin IX $\alpha$ was recovered by readjusting the $\mathrm{pH}$ to 4.0 followed by re-centrifugaton at $4500 \times g$ for $5 \mathrm{~min}$. The supernatant fluid was discarded and $20 \mathrm{~mL} \mathrm{H}_{2} \mathrm{O}$ was added to wash the mesobiliverdin IX $\alpha$ pellet. The centrifugation and washing steps were repeated twice more. Mesobiliverdin IX $\alpha(160 \mathrm{mg})$ was obtained after freeze-drying (FreeZone Plus 4.5L Cascade Benchtop Freeze Dry System, Labconco, MO, USA).

\section{BILIVERDIN IX $\alpha$}

Biliverdin IX $\alpha-\mathrm{HCl}$ was purchased from Frontier Scientific, Inc., Logan, UT (USA) and produced from recombinant E. coli (Chen et al., 2012).

\section{ANALYTICAL METHODS}

Absorbance spectra were obtained using a SpectraMax Plus384 Absorbance Microplate Reader (Molecular Devices, Sunnyvale, CA, USA). Mesobiliverdin IX $\alpha$ samples $(20 \mu \mathrm{L})$ were injected into an Alliance HPLC system (Waters, Manchester, UK) using a Symmetry ${ }^{\circledR} \mathrm{C} 18$ column $(4.6 \mathrm{~mm} \times 75 \mathrm{~mm})$ and elution gradient with solvent A (99.9\% $\mathrm{H}_{2} 0,0.1 \%$ trifluoroacetic acid) and solvent $\mathrm{B}$ (99.9\% methanol and $0.1 \%$ trifluoroacetic acid). The elution gradient program was: $100 \%$ solvent A, $1 \mathrm{~min}$; 0-60\% solvent B, 1 min; 60-100\% solvent B, 8 min, $0-100 \%$ solvent A, $1 \mathrm{~min} ; 100 \%$ solvent $\mathrm{A}, 4 \mathrm{~min}$, with a flow rate of $1 \mathrm{ml} / \mathrm{min}$. Proton NMR and two-dimensional COZY spectra of phycocyanobilin and mesobiliverdin IX $\alpha$ were collected on a Bruker AV400 with an inverse probe. For two-dimensional COZY experiments, $1024 \times 256$ data points were collected on F2 and F1, respectively, and the data were apodized with a Sinebell function and zero filled to $1 \mathrm{~K} \times 1 \mathrm{~K}$ prior to Fourier transformation. Data were processed with Mnova NMR software (Mestrelab Research, Santiago de Compostela, Spain). For mass spectroscopy, samples were analyzed on a NanoACQUITY UPLC (Waters, Manchester, UK) and a Q-Tof Primer tandem mass spectrometer (Waters, Manchester, UK). Samples $(3 \mu \mathrm{L})$ were introduced into a Symmetry ${ }^{\circledR} \mathrm{C} 18$ trapping column $(180 \mu \mathrm{m} \times 20 \mathrm{~mm})$ with NanoACQUITY Sample Manager (Waters, Manchester, UK) washed with 99\% solvent A and 1\% solvent B for $3 \mathrm{~min}$ at $15 \mu \mathrm{L} / \mathrm{min}$. Solvent A was $99.9 \% \mathrm{H}_{2} 0,0.1 \%$ formic acid and solvent B was $99.9 \%$ acetonitrile and $0.1 \%$ formic acid. Chemicals were eluted from the trapping column over a $\mathrm{BEH} 300 \mathrm{C} 4$ column with a 70 min gradient (1\% solvent B, 5 min; $1-50 \%$ solvent B, 15 min; $50-65 \%$ solvent B, 2 min; $65-85 \%$ solvent $\mathrm{B}, 21 \mathrm{~min}, 87 \%$ solvent $\mathrm{B}, 15 \mathrm{~min}, 87-1 \%$ solvent $\mathrm{B}, 3 \mathrm{~min}$, and $1 \%$ solvent, $22 \mathrm{~min}$ ) with flow rate $0.4 \mu \mathrm{L} / \mathrm{min}$. Spectral scan time was $1.0 \mathrm{~s}$.

\section{NADPH BILIVERDIN REDUCTASE ACTIVITY}

The enzymatic conversion of mesobiliverdin IX $\alpha$ to mesobilirubin was measured using the Biliverdin Reductase Assay Kit (SigmaAldrich, St. Louis, MO, USA). One mg of mesobiliverdin IX $\alpha$ was dissolved in $2 \mathrm{~mL}$ methanol, and $0.2 \mathrm{~mL}$ was mixed with $1 \mathrm{~mL}$ of the kit assay buffer. The kit-supplied recombinant human biliverdin reductase A enzyme was suspended in $800 \mu \mathrm{L}$ water, and $160 \mu \mathrm{L}$ of the enzyme suspension was added to $480 \mu \mathrm{L}$ of assay buffer. Assay buffer containing $200 \mu \mathrm{g} / \mathrm{mL}$ of mesobiliverdin $\mathrm{IX} \alpha$, E. coli produced biliverdin IX $\alpha$ or phycocyanobilin $(50 \mu \mathrm{L})$, biliverdin reductase solution $(200 \mu \mathrm{L})$, and $\mathrm{NADPH}$ solution $(0.24 \mathrm{mg} / \mathrm{mL} \mathrm{NADPH}$ in assay buffer, $750 \mu \mathrm{L})$ were combined and the absorbance spectrum between $300-800 \mathrm{~nm}$ was measured at $0,15,30,45,60,90,145,240$, and 360 min using a SpectraMax Plus384 Absorbance Microplate Reader (Molecular Devices, Sunnyvale, CA, USA).

\section{PANCREATA TREATMENT AND ISLET EQUIVALENTS}

Male Lewis rats, 300-350 g, were purchased from Charles River Laboratories, Inc. (Japan). All rats were maintained in specific pathogen-free conditions of the animal care facility and handled in accordance with institutional guidelines of the Animal Care Committee of Chiba-East National Hospital, Japan. The pancreata from rats were procured $30 \mathrm{~min}$ after dissection of inferior vena cava as a warm ischemic injury. Islets were isolated and quantitated using described procedures (Ito et al., 2010). Briefly, pancreata were distended by the infusion of Hanks' balanced salt solution supplemented with $0.1 \%$ bovine serum albumin (HBSS/BSA), $1 \mathrm{mg} / \mathrm{mL}$ of Liberase (Roche Diagnostics GmbH, Mannheim, Germany), and 1,10 , or $100 \mu \mathrm{M}$ of commercial biliverdin IX $\alpha-\mathrm{HCl}$ (Frontier Scientific, Inc., Logan, UT, USA), E. coli produced biliverdin IX $\alpha$ $\mathrm{HCl}$ (Chen et al., 2012) or mesobiliverdin IX $\alpha$. Control pancreata corresponding to each experimental test set with either commercial or E. coli produced biliverdin IX $\alpha-\mathrm{HCl}$ or mesobiliverdin IX $\alpha$ were treated with the same solution mixture but with no bile pigment. The distended and treated pancreata were incubated at $37^{\circ} \mathrm{C}$ for $30 \mathrm{~min}$. After incubation, ice-cold HBSS/BSA was added to stop enzymatic digestion. The pancreatic tissues were dissociated by repeated shaking and washing and islets were then purified by gradient centrifugation on Histopaque-1077 (Sigma-Aldrich, Japan) (Ito et al., 2010). The islets were then handpicked and the number of islets converted to the standard number of islet equivalents (IEQs) after dithizone staining (Hansen et al., 1989; Fiedor et al., 1996; Ching et al., 2001). Islet yields were expressed as IEQs/g pancreatic tissue. Photomicrographs of dithizone stained islets were obtained using a Nikon ECLIPSE TE2000-S microscope at x40 magnification. Average IEQ/g differences between groups were analyzed by the 2 -tailed unpaired Student's $t$-test and considered statistically significant when $\mathrm{p}$ values were $<0.05$.

\section{ISLET TRANSPLANTATION AND IN VIVO EVALUATION OF ENGRAFTMENT ISLET FUNCTION}

Recipient Lewis rats were made diabetic with intravenous administration of streptozotocin (STZ, $70 \mathrm{mg} \mathrm{kg}^{-1}$ ) 7 days before transplantation. Diabetes was indicated by non-fasting blood glucose (BG) levels of $>350 \mathrm{mg} / \mathrm{dL}$ in two consecutive measurements. Islets ( $\sim 500$ IEQs) isolated from donor pancreata with 30 min warm ischemia treated with or without mesobiliverdin IX $\alpha$ were infused into the portal vein of a diabetic recipient rat using a $1 \mathrm{~mL}$-capacity syringe with 25 -gage winged needle under general anesthesia. Non-fasting BG levels were measured every 2 days before and after transplantation to monitor the engraftment of islets. Reversal of diabetes was indicated when BG levels of $<200 \mathrm{mg} / \mathrm{dL}$ were determined in two consecutive measurements. To evaluate the effect of mesobiliverdin IX $\alpha$ on 
transplanted islet function, intravenous glucose tolerance tests were performed 56 post-operative days after transplantation. Under general anesthesia, test and control recipient rats were intravenously injected with $1 \mathrm{~mL} / \mathrm{kg}$ of $50 \%$ (wt/vol) glucose, and BG levels were determined at $0,2,5,10,20,30$, and $45 \mathrm{~min}$ intervals.

\section{RESULTS}

\section{MESOBILIVERDIN IX $\alpha$ PRODUCTION AND IDENTIFICATION}

Mesobiliverdin IX $\alpha$ (Figure 1C) was produced by $\mathrm{NaHCO}_{3}$ $\mathrm{K}_{2} \mathrm{CO}_{3}$-dependent isomerization of phycocyanobilin (Figure 1D) that in turn was derived and purified from dried preparations of the cyanobacterium $S$. platensis (Spirulina powder). Its identity and purity were determined by absorbance spectroscopy, TOFESI mass spectra, and two-dimensional NMR COZY analyses (Figure 2). Upon reaction with $\mathrm{K}_{2} \mathrm{CO}_{3}$ and $\mathrm{NaHCO}_{3}$, the phycocyanobilin $600 \mathrm{~nm}$ absorbance peak shifted to $640 \mathrm{~nm}$ and a $420 \mathrm{~nm}$ peak emerged (Figure 2A) indicating formation of a biliverdin-like compound. The HPLC retention time of the product was 0.07 min longer than phycocyanobilin (Figure 2B). In the phycocyanobilin two-dimensional NMR COZY spectrum, there were characteristic $=\mathrm{CH}-\mathrm{CH} 3 \mathrm{COZY}$ correlations at 6.5 and $1.9 \mathrm{ppm}$ that were absent in the product spectrum (Figure 2D) indicating the conversion of phycocyanobilin to mesobiliverdin IX $\alpha$. Its molecular mass (587.4) (Figure 2C) confirmed the identity as mesobiliverdin IX $\alpha$ and occurrence in the free acid form. Its "IX $\alpha$ " analog designation was based on structural similarities to biliverdin IX $\alpha$ and specifically the replacement of ethyl groups in place of vinyl groups in the terminal pyrrole rings of biliverdin IX $\alpha$. The mesobiliverdin IX $\alpha$ product was $>90 \%$ pure as judged by HPLC (Figure 2B) and mass spectroscopy (Figure 2C). The latter also revealed small amounts of contaminant material with molecular mass 619.5 judged to be a phycocyanobilin-methanol adduct (Beuhler et al., 1976). Yields of purified mesobiliverdin IX $\alpha$ were linearly scalable at the rate of $\sim 100 \mathrm{mg} / 160 \mathrm{~g}$ of dry Spirulina powder.

\section{MESOBILIVERDIN IX $\alpha$ AS SUBSTRATE FOR NADPH BILIVERDIN REDUCTASE}

As substrate for recombinant human NADPH bilirubin reductase, mesobiliverdin IX $\alpha$ was reduced to mesobilirubin $\left[\lambda_{\max }\right.$, $440 \mathrm{~nm}$ (Terry et al., 1993)] at rates that were equivalent to those for catalytic conversion of biliverdin IX $\alpha$ to bilirubin IX $\alpha$ $\left(\lambda_{\max }, 460 \mathrm{~nm}\right)$ (Figure 3 ). In contrast, phycocyanobilin, the synthetic precursor to mesobiliverdin IX $\alpha$, was a relatively poor

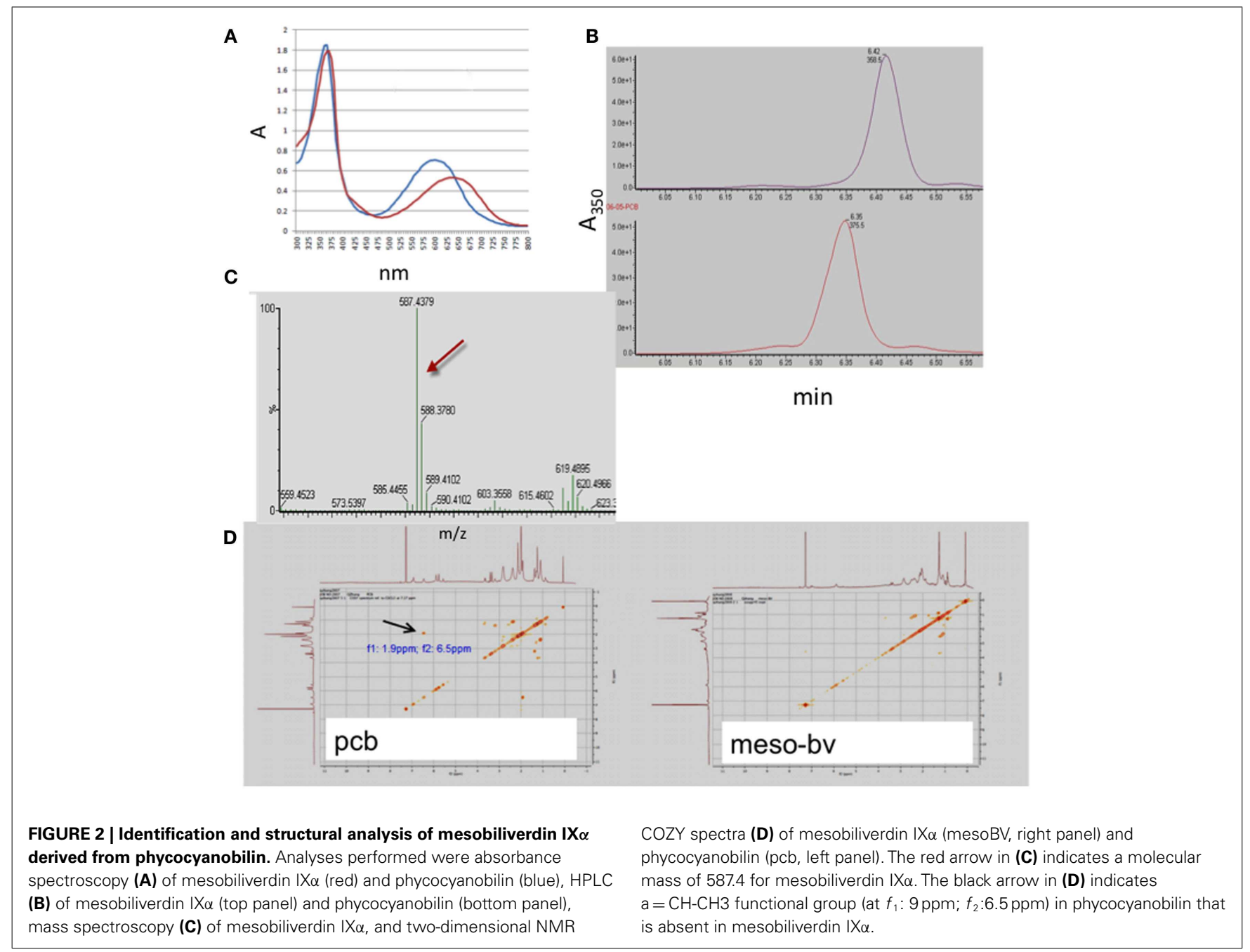




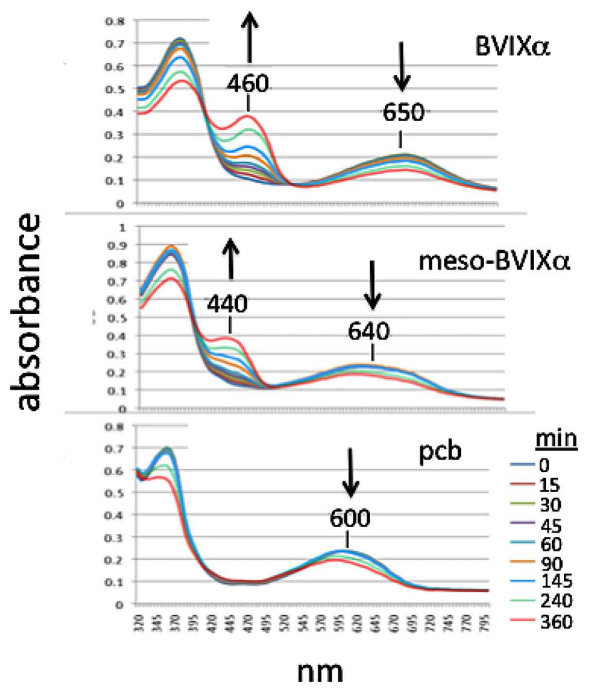

FIGURE 3 |Time-course of reactions catalyzed by human recombinant NADPH biliverdin reductase with $E$. coli produced biliverdin $\mathrm{IX} \alpha-\mathrm{HCl}$ (BVIX $\alpha$ ), mesobiliverdin IX $\alpha$ (meso-BVIX $\alpha$ ), and phycocyanobilin (pcb) as substrates. $\mathrm{NADPH}$-dependent reduction was monitored spectrophotometrically for $6 \mathrm{~h}$.

substrate as judged by the inability to detect catalytic conversion to phycocyanorubin $\left[\lambda_{\max }, 420 \mathrm{~nm}\right.$ (Terry et al., 1993)].

\section{EFFECT ON PANCREATIC ISLET YIELD AND VIABILITY}

Pancreata were infused with HBSS/BSA solutions containing mesobiliverdin IX $\alpha$, commercial biliverdin IX $\alpha-\mathrm{HCl}$ or $E$. coli produced biliverdin IX $\alpha-\mathrm{HCl}$, followed by islet isolation and determination of IEQs/g yields. Solutions containing mesobiliverdin IX $\alpha$ (at $1-100 \mu \mathrm{M}$ ) yielded IEQ/g increases ranging between 54 and $86.7 \%$ over controls (without mesobiliverdin IX $\alpha$ ) (Table 1). The highest average IEQ/g (86.7\% over controls) was achieved with $1 \mu \mathrm{M}$ mesobiliverdin IX $\alpha$. Infusion with solutions containing commercial biliverdin IX $\alpha-\mathrm{HCl}$ gave IEQ/g average increases as high as $35.5 \%$ (at $10 \mu \mathrm{M}$ ) over controls and with recombinant E. coli produced biliverdin IX $\alpha-\mathrm{HCl}$, as high as $36.5 \%$ (at $100 \mu \mathrm{M})$. High degrees of islet viabilities as judged by dithizone staining were observed with mesobiliverdin IX $\alpha$ and $E$. coli produced biliverdin IX $\alpha-\mathrm{HCl}$ and a comparatively lower degree of viability was observed with no treatment (Figure 4).

\section{RECIPIENT IN VIVO ISLET FUNCTION AFTER TREATMENT OF DONOR PANCREATA WITH MESOBILIVERDIN IX $\alpha$}

Islets ( $\sim 500$ IEQs) obtained as described above with or without mesobiliverdin IX $\alpha(100 \mu \mathrm{M})$ treatment were transplanted by infusion into recipient livers of STZ induced diabetic rats through the portal vein. Subsequent recipient BG levels revealed diabetes reversal in $55.6 \%$ (five of nine) of the rats receiving islets from mesobiliverdin IX $\alpha 100 \mu \mathrm{M}$ ) - treated pancreata (Figure 5 lower panel); $22.2 \%$ (two of nine) of the non-treated control recipients showed diabetes reversal (Figure 5 upper panel). Intravenous glucose tolerance tests on day 56 also revealed improved islet function with mesobiliverdin IX $\alpha$ infusion of donor pancreata.
Table 1 | Islet yields from pancreata infused with biliverdin IX $\alpha-\mathrm{HCl}$ and mesobiliverdin IX $\alpha$.

\begin{tabular}{|c|c|c|c|}
\hline Treatment $^{1}$ & $\begin{array}{l}\text { IEQs g }{ }^{-1} \text { (average, } \\
\text { std, range, no. of values) }\end{array}$ & $P$ value & $\begin{array}{l}\% \text { above } \\
\text { control }\end{array}$ \\
\hline $1 \mu \mathrm{MBV} \mathrm{FS}^{2}$ & $1328 \pm 358(591-1705)(8)$ & 0.426 & 11.3 \\
\hline $10 \mu \mathrm{MBV} F$ & $1617 \pm 451$ (1006-2519) (8) & 0.037 & 35.5 \\
\hline $100 \mu \mathrm{M} \mathrm{BV} F$ & $1527 \pm 403(942-2363)(9)$ & 0.050 & 28.0 \\
\hline Control & $1193 \pm 223(931-1307)(9)$ & & \\
\hline $1 \mu \mathrm{MBV} \mathrm{EC}^{3}$ & $1345 \pm 629(662-2234)(7)$ & 0.860 & 4.3 \\
\hline $10 \mu \mathrm{M} \mathrm{BV} \bigvee_{E C}$ & $1603 \pm 1073(901-4117)(8)$ & 0.480 & 24.4 \\
\hline $100 \mu \mathrm{M} \mathrm{BV} V_{E C}$ & $1759 \pm 703(658-2593)(8)$ & 0.163 & 36.5 \\
\hline Control & $1289 \pm 559(579-2182)(8)$ & & \\
\hline $1 \mu \mathrm{M}$ mesoBV 4 & $1599 \pm 475(1004-2053)(7)$ & 0.005 & 86.7 \\
\hline $10 \mu \mathrm{M}$ mesoBV & $1318 \pm 805(655-2946)(8)$ & 0.156 & 54.0 \\
\hline $100 \mu \mathrm{M}$ mesoBV & $1535 \pm 287(1145-1923)$ & 0.0002 & 79.3 \\
\hline Control & $856 \pm 229$ (539-1166) (8) & & \\
\hline $20 \mu \mathrm{M}$ p38IH $\mathrm{H}^{5}$ & $2134 \pm 297(997-2837)$ & 0.037 & 45.1 \\
\hline Control $^{5}$ & $1477 \pm 145(1118-1889)$ & 0.037 & \\
\hline
\end{tabular}

${ }^{1}$ Seven to nine organs per infusion treatment.

${ }^{2}$ Biliverdin IX $\mathrm{X}-\mathrm{HCl}$ purchased from Frontier Scientific, Inc., Logan, UT, USA.

${ }^{3}$ Biliverdin IXa-HCl produced by recombinant $E$. coli as previously described (Chen et al., 2012).

${ }^{4}$ Mesobiliverdin IXa (this work).

${ }^{5}$ p38 MAPkinase inhibitor; data from canine experiments (six organs per infusion treatment) reported in Ito et al. (2008).

Fasting BG levels measured at zero and 2 min indicated recovery of insulin-dependent function and were significantly lower with transplanted islets from pancreata treated with mesobiliverdin IX $\alpha$ as compared to controls with islets from non-treated pancreata (Figure 6).

\section{DISCUSSION}

Growing evidence suggests the therapeutic potential of biliverdin IX $\alpha$ against acute and chronic inflammatory conditions such as diabetes (Florczyk et al., 2008; Wang et al., 2011; Gibbs et al., 2012; Wegiel and Otterbein, 2012). Its current development as an anti-inflammatory pharmaceutical, however, is hampered by its commercial scarcity, contamination by isomers, and derivation from animal sources. The recently reported scalable production of biliverdin IX $\alpha$ by E. coli represents an attempt to address these issues (Chen et al., 2012). With the goal of producing a pharmaceutical equivalent of biliverdin IX $\alpha$ that also overcomes these limitations, the production of the mesobiliverdin IX $\alpha$ from a non-animal source (i.e., cyanobacteria) was developed. The synthesis of mesobiliverdin IX $\alpha$ from phycocyanobilin facilitated by $\mathrm{NaHCO}_{3}-\mathrm{K}_{2} \mathrm{CO}_{3}$ is efficient, as is the methanolic cleavage of phycocyanobilin from the apoprotein of phycocyanin. Phycocyanin itself is an abundant, water-soluble pigment-protein complex that in turn is easily extracted from photosynthetic microbes of the groups cyanobacteria, rhodophyta, and cryptophyta. Hence, the procedure is scalable for the production of large quantities of mesobiliverdin IX $\alpha$. 


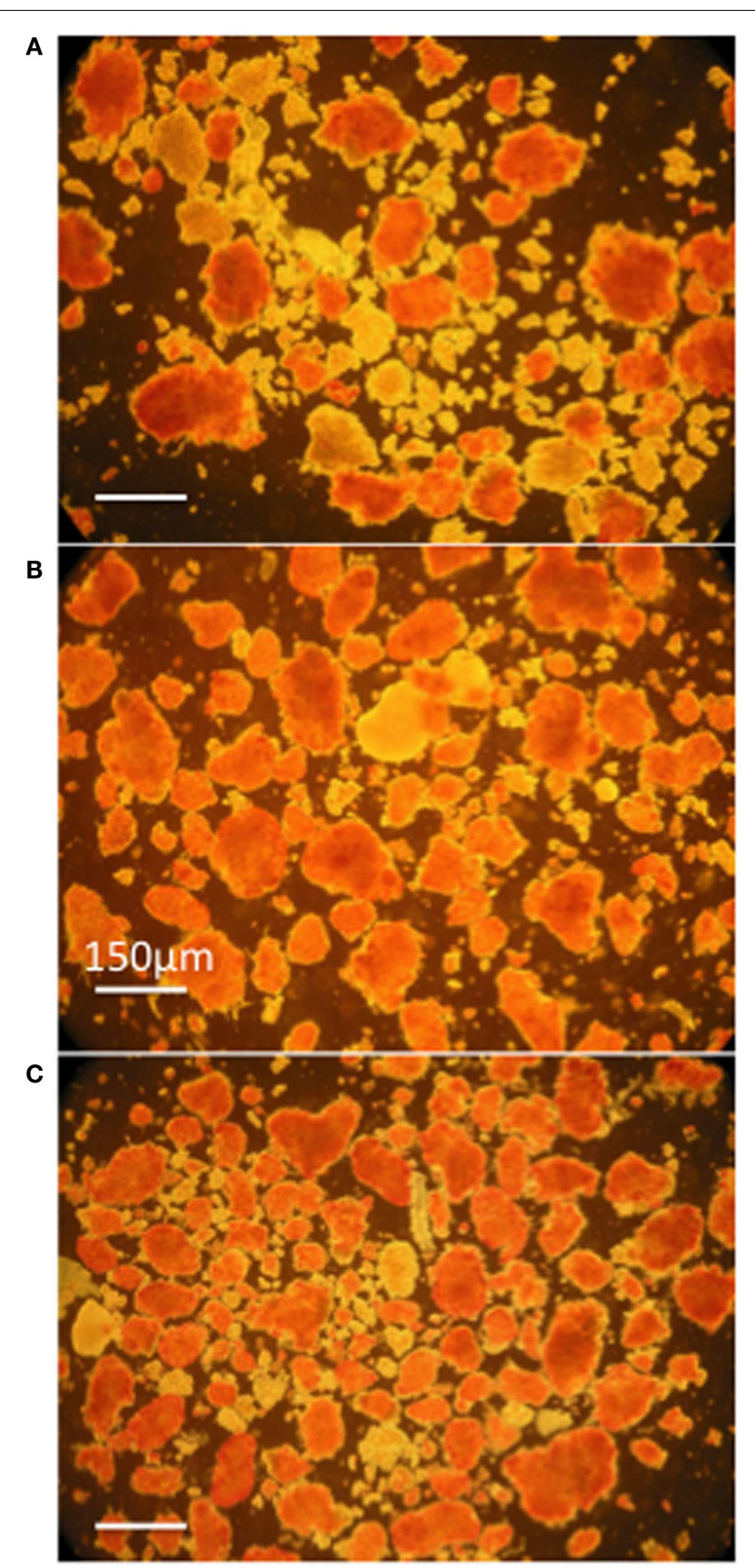

FIGURE 4 | Photomicrographs of dithizone stained islet preparations derived from donor Lewis rat pancreata infused with HBSS/BSA solution (A) and HBSS/BSA solution containing $100 \mu \mathrm{M}$ mesobiliverdin IX $\alpha$ (B) or $\mathbf{1 0 0} \mu \mathrm{M}$ E. coli produced biliverdin IX $\alpha-\mathrm{HCl}$ (C). Viable islets are stained red. The bar designates a length of $150 \mu \mathrm{m}$.

Mesobiliverdin IX $\alpha$ differs from biliverdin IX $\alpha$ by the replacement of vinyl groups with ethyl groups at positions 3 and 18 of the linear tetrapyrrole structure (Figure 1). The differences are not expected to significantly affect substrate specificity binding to the active site of human biliverdin reductase for catalytic reduction to the corresponding product (i.e., mesobilirubin or bilirubin

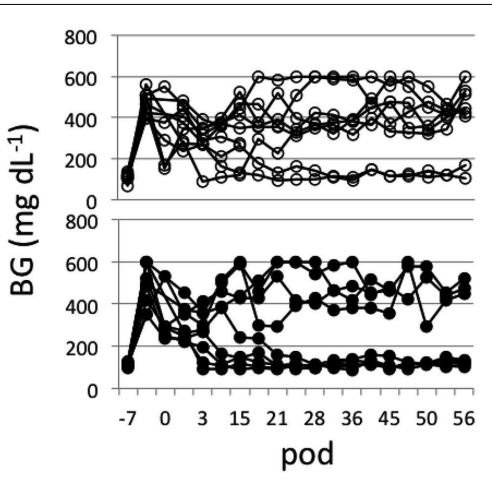

FIGURE 5 | Non-fasting blood glucose (BG) level profiles of STZ induced diabetic Lewis rats measured for up to 56 post-operative days (pod) following transplantation with islets ( 500 IEQs) from donor pancreata infused with HBSS/BSA solution containing $100 \mu \mathrm{M}$ mesobiliverdin IX $\alpha(n=9)$ (lower panel) or without mesobiliverdin IX $\alpha$ $(n=9)$ (upper panel).

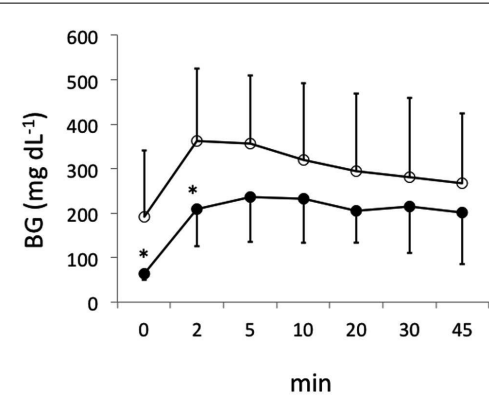

FIGURE 6 | Fasting blood glucose (BG) levels following intravenous tolerance tests of STZ induced diabetic Lewis rats. Tests were performed on day 56 following transplantation with islets from donor pancreata infused with HBSS/BSA solution containing $100 \mu \mathrm{M}$ mesobiliverdin IX $\alpha(n=7)$ (filled circles) or without mesobiliverdin IX $\alpha$ ( $n=9$ ) (open circles). BG levels at 0 and 2 min correlated with first-phase insulin function. ${ }^{*} p$ values $<0.05$.

IX $\alpha$ ) (Cunningham et al., 2000; Fu et al., 2012) as supported in the present study (Figure 3). Substrate binding to biliverdin reductase appears central to the downstream anti-inflammatory and anti-pro-inflammatory effects of biliverdin IX $\alpha$ (Gibbs et al., 2012; Wegiel and Otterbein, 2012). These considerations suggest that mesobiliverdin IX $\alpha$ may have therapeutic effects similar to those shown experimentally for biliverdin IX $\alpha$ in numerous animal studies. In comparison to mesobiliverdin IX $\alpha$ and biliverdin IX $\alpha$, phycocyanobilin appeared to be a weaker substrate for NADPH biliverdin reductase (Figure 3) suggesting that it may have less effective anti-inflammatory capabilities (Zheng et al., 2012).

Both biliverdin IX $\alpha$ and mesobiliverdin IX $\alpha$ had protective effects against islet degradation following pancreatectomy. The degree of protection by mesobiliverdin IX $\alpha$ exceeded those provided by biliverdin IX $\alpha$ (Table 1). Pancreatic infusion with as low as $1 \mu \mathrm{M}$ mesobiliverdin IX $\alpha$ gave nearly twofold higher IEQs/g than 10 and $100 \mu \mathrm{M}$ biliverdin IX $\alpha$ and p38 MAPkinase inhibitor previously observed in canine islet transplant experiments (Table 1) (Ito 
et al., 2008). Mesobiliverdin IX $\alpha$ at $1 \mu \mathrm{M}$ gave an average $86.7 \%$ increase in IEQs/g over non-treatment controls. This degree of improvement in islet yield is clinically significant since currently two or more pancreatic donor organs are required per recipient to achieve insulin independence (Shapiro et al., 2005; Ito et al., 2008; Wang et al., 2011).

In STZ induced diabetic rat transplantation experiments, mesobiliverdin IX $\alpha$ infusion with 30 min warm ischemic injury improved graft function of rat islets (Figures 5 and 6). Changes in fasting BG levels at 0 and $2 \mathrm{~min}$ in intravenous glucose tolerance tests indicated recovery of insulin-dependent function against a glucose load, and the overall levels were significantly lower than controls receiving islets from untreated pancreata. Thus improved yields of functional islets were achieved with mesobiliverdin IX $\alpha$ infusion of donor pancreata.

Why mesobiliverdin IX $\alpha$ at lower concentration $(1 \mu \mathrm{M})$ protected better than commercial biliverdin IX $\alpha$ and E. coli produced biliverdin IX $\alpha$ is not known. Reasons may lie in differences of their chemical state and purity. The biliverdin IX $\alpha$ preparations used were of the hydrochloride form whereas the mesobiliverdin IX $\alpha$ was produced as the free acid-differences

\section{REFERENCES}

Bellner, L., Wolstein, J., Patil, K. A., Dunn, M. W., and LaniadoSchwartzman, M. (2011). Biliverdin rescues the HO-2 null mouse phenotype of unresolved chronic inflammation following corneal epithelial injury. Invest. Ophthalmol. Vis. Sci. 52, 3246-3253.

Beuhler, R. J., Pierce, R. C., Friedman, L., and Siegelman, H. W. (1976). Cleavage of phycocyanobilin from $C$ phycocyanin. Separation and mass spectral identification of the products. J. Biol. Chem. 251, 2405-2411.

Chen, D., Brown, J. D., Kawasaki, Y., Bommer, J., and Takemoto, J. Y. (2012). Scalable production of biliverdin IXalpha by Escherichia coli. BMC Biotechnol. 12:89. doi:10.1186/1472-6750-12-89

Ching, C. D., Harland, R. C., Collins, B. H., Kendall, W., Hobbs, H., and Opara, E. C. (2001). A reliable method for isolation of viable porcine islet cells. Arch. Surg. 136, 276-279.

Cunningham, O., Dunne, A., Sabido, P., Lightner, D., and Mantle, T. J. (2000). Studies on the specificity of the tetrapyrrole substrate for human biliverdin-IXalpha reductase and biliverdin-IXbeta reductase. Structure-activity relationships define models for both active sites. J. Biol. Chem. 275, 19009-19017.

Emamaullee, J. A., and Shapiro, A. M. J. (2006). Interventional strategies to prevent beta-cell apoptosis in islet transplantation. Diabetes 55, 1907-1914.

Fiedor, P., Walaszewski, J., Oluwole, S. F., Licinska, I., Mazurek, A. P., Hardy,
M. A., et al. (1996). A novel approach to in vivo visualization of human pancreatic islets. Transplant. Proc. $28,3514$.

Florczyk, U. M., Jozkowicz, A., and Dulak, J. (2008). Biliverdin reductase: new features of an old enzyme and its potential therapeutic significance. Pharmacol. Rep. 60, 38-48.

Fondevila, C., Katori, M., Lassman, C., Carmody, I., Busuttil, R. W., Bach, F. H., et al. (2003). Biliverdin protects rat livers from ischemia/reperfusion injury. Transplant. Proc. 35, 1798-1799.

Fu, G., Liu, H., and Doerksen, R. J. (2012). Molecular modeling to provide insight into the substrate binding and catalytic mechanism of human biliverdin-IXalpha reductase. J. Phys. Chem. B 116, 9580-9594.

Fujii, M., Inoguchi, T., Sasaki, S., Maeda, Y., Zheng, J., Kobayashi, K., et al. (2010). Bilirubin and biliverdin protect rodents against diabetic nephropathy by downregulating $\mathrm{NAD}(\mathrm{P}) \mathrm{H}$ oxidase. Kidney Int. 78, 905-919.

Gibbs, P. E., Tudor, C., and Maines, M. D. (2012). Biliverdin reductase: more than a namesake the reductase, its peptide fragments, and biliverdin regulate activity of the three classes of protein kinase C. Front. Pharmacol. 3:31. doi:10.3389/fphar.2012.00031

Gibbs, P. E. M., and Maines, M. D. (2007). Biliverdin inhibits activation of NF-кB: reversal of inhibition by human biliverdin reductase. Int. J. Cancer 121, 2567-2574.

that could have bearing on tissue and cell accessibility. Commercial biliverdin IX $\alpha$ preparations derived from animal sources and produced from conjugated bilirubin often contain inactive isomers (McDonagh, 2005). The E. coli derived biliverdin IX $\alpha$ could possibly contain lipopolysaccharide endotoxin that would compromise its anti-inflammatory capabilities. Alternatively, the more cytoprotective effect of mesobiliverdin IX $\alpha$ may result from as yet unknown variations of the antiinflammatory mechanisms of this bioactive porphyrin. Further investigations are needed to better understand the cytoprotective mechanisms of mesobiliverdin IX $\alpha$ in comparison to those for biliverdin IX $\alpha$ and other anti-inflammatory heme derived porphyrins.

\section{ACKNOWLEDGMENTS}

NMR COZY analyses by Dr. Zhicheng Xia (University of British Columbia, British Columbia, Canada) are gratefully acknowledged. This research was supported by the Utah Science, Technology and Research (USTAR) Initiative, State of Utah, and the Synthetic Bioproducts Institute, Utah State University, Logan, UT, USA.

Greenberg, A. J., Bossenmaier, I., and Schwartz, S. (1971). Green jaundice. A study of serum biliverdin, mesobiliverdin and other green pigments. Am. J. Dig. Dis. 16, 873-880.

Hansen, W., Christie, M. R., Kahn, R., Norgaard, A., Abel, I., Petersen, A. M., et al. (1989). Supravital dithizone staining in the isolation of human and rat pancreatic islets. Diabetes Res. 10, 53-57.

Ikeda, N., Inoguchi, T., Sonoda, N. Fujii, M., Takei, R., Hirata, E., et al. (2011). Biliverdin protects against the deterioration of glucose tolerance in $\mathrm{db} / \mathrm{db}$ mice. Diabetologia 54 , 2183-2191.

Ito, T., Itakura, S., Todorov, I., Rawson, J., Asari, S., Shintaku, J., et al. (2010). Mesenchymal stem cell and islet co-transplantation promotes graft revascularization and function. Transplantation 89, 1438-1445.

Ito, T., Omori, K., Rawson, J., Todorov, I., Asari, S., Kuroda, A., et al. (2008). Improvement of canine islet yield by donor pancreas infusion with a p38MAPK inhibitor. Transplantation 86, 321-329.

Kapitulnik, J., and Maines, M. D. (2012). The role of bile pigments in health and disease: effects on cell signaling, cytotoxicity and cytoprotection. Front. Pharmacol. 3:136. doi:10.3389/fphar.2012.00136

Kenmochi, T., Maruyama, M., Saigo, K., Akutsu, N., Iwashita, C., Otsuki, K., et al. (2008). Successful islet transplantation from the pancreata of non-heart-beating donors. Transplant. Proc. 40, 2568-2570.

Matsumoto, S., Noguchi, H., Naziruddin, B., Onaca, N., Jackson, A.
Nobuyo, H., et al. (2007). Improvement of pancreatic islet cell isolation for transplantation. Proc. (Bayl. Univ. Med. Cent.) 20, 357-362.

McDonagh, A. F. (2001). Turning green to gold. Nat. Struct. Biol. 8, 198-200.

McDonagh, A. F. (2005). Biliverdin, immune-mediated liver injury, and the gigo effect. Hepatology 41, 680-681.

McPhee, F., Caldera, P. S., Bemis, G. W., Mcdonagh, A. F., Kuntz, I. D., and Craik, C. S. (1996). Bile pigments as HIV-1 protease inhibitors and their effects on HIV-1 viral maturation and infectivity in vitro. Biochem. J. 320, 681-686.

Najarian, J. S., Sutherland, D. E., Matas, A. J., Steffes, M. W., Simmons, R. L., and Goetz, F. C. (1977). Human islet transplantation: a preliminary report. Transplant. Proc. 9, 233-236.

Nakagami, T., Taji, S., Takahashi, M., and Yamanishi, K. (1992). Antiviral activity of a bile pigment, biliverdin, against human herpesvirus 6 (HHV6) in vitro. Microbiol. Immunol. 36, 381-390.

Nakao, A., Murase, N., Ho, C., Toyokawa, H., Billiar, T. R., and Kanno, S. (2005). Biliverdin administration prevents the formation of intimal hyperplasia induced by vascular injury. Circulation 112, 587-591.

Nakao, A., Otterbein, L. E., Overhaus, M., Sarady, J. K., Tsung, A., Kimizuka, K., et al. (2004). Biliverdin protects the functional integrity of a transplanted syngeneic small bowel. Gastroenterology 127, 595-606. 
Ollinger, R., Bilban, M., Erat, A., Froio, A., Mcdaid, J., Tyagi, S., et al. (2005). Bilirubin: a natural inhibitor of vascular smooth muscle cell proliferation. Circulation 112, 1030-1039.

Overhaus, M., Moore, B. A., Barbato, J. E., Behrendt, F. F., Doering, J. G., and Bauer, A. J. (2006). Biliverdin protects against polymicrobial sepsis by modulating inflammatory mediators. Am. J. Physiol. Gastrointest. Liver Physiol. 290, G695-703.

Pachori, A. S., Smith, A., Mcdonald, P., Zhang, L., Dzau, V. J., and Melo, L. G. (2007). Heme-oxygenase1-induced protection against hypoxia/reoxygenation is dependent on biliverdin reductase and its interaction with PI3K/Akt pathway. J. Mol. Cell. Cardiol. 43, 580-592.

Ribeiro, M. M., Klein, D., Pileggi, A., Molano, R. D., Fraker, C., Ricordi, C., et al. (2003). Heme oxygenase1 fused to a TAT peptide transduces and protects pancreatic betacells. Biochem. Biophys. Res. Commun. 305, 876-881.

Ryan, E. A., Paty, B. W., Senior, P. A., and Shapiro, A. M. (2004). Risks and side effects of islet transplantation. Curr. Diab. Rep. 4, 304-309.

Sarady-Andrews, J. K., Liu, F., Gallo, D., Nakao, A., Overhaus, M., Ollinger, R., et al. (2005). Biliverdin administration protects against endotoxininduced acute lung injury in rats. Am. J. Physiol. Lung Cell. Mol. Physiol. 289, L1131-L1137.

Sedlak, T. W., and Snyder, S. H. (2004). Bilirubin benefits: cellular protection by a biliverdin reductase antioxidant cycle. Pediatrics 113 , 1776-1782.

Shapiro, A. M., Lakey, J. R., Paty, B. W. Senior, P. A., Bigam, D. L., and Ryan, E. A. (2005). Strategic opportunities in clinical islet transplantation. Transplantation 79, 1304-1307.

Shapiro, A. M. J., Lakey, J. R. T., Ryan, E. A., Korbutt, G. S., Toth, E., Warnock, G. L., et al. (2000). Islet transplantation in seven patients with type 1 diabetes mellitus using a glucocorticoid-free immunosuppressive regimen. N. Eng. J. Med.343, 230-238.

Soares, M. P., and Bach, F. H. (2009). Heme oxygenase-1: from biology to therapeutic potential. Trends Mol. Med. 15, 50-58.

Stocker, R., Yamamoto, Y., Mcdonagh, A., Glazer, A., and Ames, B. (1987). Bilirubin is an antioxidant of possible physiological importance. Science 235, 1043-1046.

Terry, M. J., Maines, M. D., and Lagarias, J. C. (1993). Inactivation of phytochrome- and phycobiliprotein-chromophore precursors by rat liver biliverdin reductase. J. Biol. Chem. 268, 26099-26106.

Tiribelli, C., and Ostrow, J. D. (2005). Intestinal flora and bilirubin. $J$. Hepatol. 42, 170-172.

Vitek, L., Majer, F., Muchova, L., Zelenka, J., Jiraskova, A., Branny, P., et al. (2006). Identification of bilirubin reduction products formed by Clostridium perfringens isolated from human neonatal fecal flora. J. Chromatogr. B Analyt. Technol. Biomed. Life Sci. 833, 149-157.

Wang, H., Ferran, C., Attanasio, C., Calise, F., and Otterbein, L. E. (2011). Induction of protective genes leads to islet survival and function. $J$. Transplant. 2011, 141898.

Wegiel, B., Baty, C. J., Gallo, D., Csizmadia, E., Scott, J. R., Akhavan, A., et al. (2009). Cell surface biliverdin reductase mediates biliverdin-induced anti-inflammatory effects via phosphatidylinositol 3-kinase and Akt. $J$. Biol. Chem. 284, 21369-21378.

Wegiel, B., and Otterbein, L. (2012). Go green: the antiinflammatory effects of biliverdin reductase. Front. Pharmacol. 3:47. doi:10.3389/fphar.2012.00047

Yamashita, K., Mcdaid, J., Ollinger, R. Tsui, T.-Y., Berberat, P. O., Usheva, A., et al. (2004). Biliverdin, a natural product of heme catabolism, induces tolerance to cardiac allografts. FASEB J. 18, 765-767.

Zheng, J., Inoguchi, T., Sasaki, S., Maeda, Y., Mccarty, M., Fujii, M., et al. (2012). Phycocyanin and phycocyanobilin from Spirulina platensis protect against diabetic nephropathy by inhibiting oxidative stress. Am. J. Physiol. Regul. Comp. Physiol 304, R110-R120.

Zhou, H., Qian, H., Liu, J., Zhu, D., Ding, W., Pan, P., et al. (2011). Protection against lung graft injury from braindead donors with carbon monoxide, biliverdin, or both. J. Heart Lung Transplant. 30, 460-466.
Zhu, Z., Wilson, A. T., Luxon, B. A., Brown, K. E., Mathahs, M. M., Bandyopadhyay, S., et al. (2010). Biliverdin inhibits hepatitis $\mathrm{C}$ virus nonstructural 3/4A protease activity: mechanism for the antiviral effects of heme oxygenase? Hepatology 52, 1897-1905.

Conflict of Interest Statement: The authors declare that the research was conducted in the absence of any commercial or financial relationships that could be construed as a potential conflict of interest.

Received: 12 February 2013; paper pending published: 17 March 2013; accepted: 31 March 2013; published online: 23 April 2013.

Citation: Ito T, Chen D, Chang C-WT, Kenmochi T, Saito T, Suzuki S and Takemoto JY (2013) Mesobiliverdin IXo enhances rat pancreatic islet yield and function. Front. Pharmacol. 4:50. doi: 10.3389/fphar.2013.00050

This article was submitted to Frontiers in Drug Metabolism and Transport, a specialty of Frontiers in Pharmacology. Copyright (c) 2013 Ito, Chen, Chang, Kenmochi, Saito, Suzuki and Takemoto. This is an open-access article distributed under the terms of the Creative Commons Attribution License, which permits use, distribution and reproduction in other forums, provided the original authors and source are credited and subject to any copyright notices concerning any third-party graphics etc. 